\title{
Factores Familiares asociados a la presencia de Caries Dental en Niños Escolares de Cartagena, Colombia
}

\author{
Shyrley Díaz Càrdenas ${ }^{a}$, Katherine Arrieta Vergaraa ${ }^{a}$ Farith González Martinez
}

\begin{abstract}
a Odontóloga, Magíster en Salud Pública, Departamento de Odontología Preventiva y Social, Facultad de Odontología, Universidad de Cartagena, Colombia.

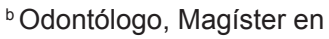
Salud Pública, Departamento de Investigación, Facultad de Odontología, Universidad de Cartagena, Colombia.
\end{abstract}

Correspondencia: Shyrley Díaz Cárdenas, Universidad de Cartagena, Campus de la Salud Zaragocilla, Facultad de Odontología, Departamento de Investigaciones. Telf.: 6698184 ext. 110, 3176355324, e-mail: shyrley77@hotmail.com.

Recibido el 28 de diciembre de 2010.

Aceptado para su publicación el 18 de marzo de 2011.

\section{RESUMEN}

Objetivo. Identificar los factores socios demográficos y familiares y su asociación con la prevalencia de caries en niños del colegio Madre Gabriela de San Martín.

Diseño. Estudio de corte transversal.

Emplazamiento. Municipio de Cartagena de Indias-Colombia.

Participantes. 243 estudiantes seleccionados aleatoriamente por muestreo estratificado.

Mediciones Principales. Se evaluaron factores socio demográficos y familiares (unión conyugal, escolaridad y rol de los padres, estrato socioeconómico estructura y funcionalidad familiar mediante APGAR familiar) y prevalencia de caries (índice COP-D y ceo-d). Los datos fueron analizados a partir de proporciones y razones de disparidad utilizando el programa Stata 10.0.

Resultados. La prevalencia de caries fue de 51\% (IC 95\%: 44-57), más en el sexo femenino $(70 \% ; p=0,02)$. Al ser relacionadas las variables familiares con la caries dental, únicamente se encontró asociación significativa con el rol ejercido por el padre como formador de hijos (OR: 0,40; IC 95\%: 0,18-0,86; $p<0,01$ ).

Conclusiones. Además de ser tradicionalmente un proveedor económico, el nuevo rol del padre como formador de hijo puede influir positivamente en la disminución de la caries dental y debe ser parte activa en las actividades de promoción y prevención en salud bucal.

Palabras Claves. Caries Dental, Medicina Familiar y Comunitaria, Niños.

\section{ABSTRACT}

Family factors associated with the prevalence of caries in school children in Cartagena, Colombia

Objective. To identify sociodemographic and family factors and their association with the prevalence of caries in children attending the Madre Gabriela de San Martín.

Design. Cross sectional study.

Setting. Cartagena de Indias, Colombia.

Participants. 243 students randomly selected by stratified sampling.

Main Measurements. Sociodemographic and family factors (marital relationship, education and role of parents, socioeconomic status and family structure and function using the Family APGAR) and prevalence of caries (COP-D index and ceo-d). The data were analyzed based on proportions and odds ratio using the Stata 10.0 programme

Results. The prevalence of caries was $51 \%$ (95\% Cl: $44-57)$, higher in females $(70 \% ; p=0.02)$. The role of the father as educator of his children was the only significant factor (OR: $0.40 ; 95 \%$ Cl: 0.18 to $0.86 ; p<0.01$ ) related to the prevalence of caries.

Conclusions. In addition to being traditionally an economic provider, the new role of the father as educator of his children can have a positive effect on reducing dental caries. Fathers should be actively involved in the promotion and prevention activities in oral health.

Key words. Dental Caries, Family and Community Medicine, Child.

\section{INTRODUCCIÓN}

La salud bucal de los niños se inicia desde el hogar ${ }^{1}$. Se han relacionado los conocimientos, actitudes y prácticas de salud bucal de los padres con el estado de salud bucal de sus hijos ${ }^{2}$. También se han relacionado la baja escolaridad y el hecho de no tener empleo los padres ${ }^{3}$, pertenecer a estrato socioeconómico bajo ${ }^{4}$, la delegación del cuidado de los niños a abuelos 
o cuidadores por largas jornadas de trabajo de las madres ${ }^{5}$, problemas de salud bucal de los miembros de las familias ${ }^{6}$, tener más de 4 hijos $^{3}$ y el abandono físico ${ }^{7}$ como factores familiares de riesgo para la caries dental. La disfuncionalidad y el tipo de estructura familiar, así como las familias monoparentales, podrían incrementar el riesgo de sufrir caries dental y su severidad ${ }^{8}$.

En Colombia poco se ha estudiado al respecto y actualmente en Cartagena hay estudios en escolares que reportan relaciones entre la caries dental y factores familiares, como el realizado por González en 2004, pero no establecen estos últimos como posibles productores de riesgo para caries dental $^{9}$, lo que implica que no se puedan tomar medidas que contribuyan a controlar o evitar estos factores de riesgo desde el diseño de los programas de promoción y prevención implementados. La familia y la comunidad juegan un papel importante en la promoción de la salud bucal y la prevención de enfermedades, pero se necesitan familias funcionales y bien estructuradas que contribuyan en este proceso formador, a través del abordaje integral y del rescate de la familia como el primer núcleo socializador del individuo.

El objetivo de este estudio fue identificar los factores de riesgo socios demográficos y familiares y su asociación con la prevalencia de caries en niños preescolares y escolares del colegio Madre Gabriela de San Martín.

\section{MATERIAL Y MÉTODO}

Estudio de corte transversal, realizado en la institución educativa oficial Madre Gabriela de San
Martín. La población marco la constituyen 661 niños que cursan preescolar y básica primaria. El tamaño de la muestra fue de 243 sujetos, calculado a partir de una prevalencia esperada de caries dental del 55 $\%^{2}$, nivel de confianza del $95 \%$, error permitido de 0,05 y un poder del $80 \%$. El proceso de selección se realizó en forma probabilística estratificada, a partir de una fijación proporcional por el grado de escolaridad de los niños. Como criterios de exclusión se tuvieron en cuenta las enfermedades sistémicas como diabetes, hipertensión, síndrome de Down o cualquier tipo de discapacidad motora y sensorial.

Para el inicio de las mediciones se solicitó el consentimiento informado por escrito de la institución educativa y de los padres de los niños, teniendo en cuenta la resolución 8430 de 1993 del Ministerio de Salud de la República de Colombia.

Se utilizó un instrumento clínico, basado en el formato Guías de Práctica Clínica en salud oral de la Secretaria Distrital de Salud, Bogotá-ACFO, 2006, aplicándose el índice de COP para la prevalencia de experiencia de caries y tomando como parámetro un valor igual o mayor a dos. Este diagnóstico se llevó a cabo en los consultorios odontológicos ubicados en el interior del colegio. Posteriormente, se realizaron visitas domiciliarias para la medición de las características socio demográficas y familiares, como el estrato socioeconómico, estado civil, composición familiar, estructura y funcionalidad familiar (APGAR familiar ${ }^{1}$ ), utilizando un cuestionario con 32 preguntas estructuradas con una sola opción de respuesta.

\begin{tabular}{|c|c|c|c|c|c|c|}
\hline \multirow[b]{2}{*}{ Rango Edad } & \multicolumn{2}{|c|}{ Total $(n=243)$} & \multicolumn{2}{|c|}{ Niños $(n=123)$} & \multicolumn{2}{|c|}{ Niñas $(n=120)$} \\
\hline & $\mathbf{N}^{\circ}$ & $(\%)$ & $\mathbf{N}^{\circ}$ & $(\%)$ & $\mathbf{N}^{0}$ & $(\%)$ \\
\hline 4 a 5 & 41 & 17 & 18 & 15 & 23 & 19 \\
\hline 6 a 7 & 66 & 27 & 28 & 23 & 38 & 32 \\
\hline 8 a 9 & 74 & 30 & 38 & 31 & 36 & 30 \\
\hline 10 a 11 & 55 & 23 & 36 & 29 & 19 & 16 \\
\hline 12 a 13 & 7 & 3 & 3 & 2 & 4 & 3 \\
\hline Total & 243 & 100 & 123 & 100 & 120 & 100 \\
\hline
\end{tabular}

Tabla 1. Población objeto por edad y sexo en la Institución Educativa Madre Gabriela de San Martín. 2010. 


\begin{tabular}{lccc}
\hline Variables Familiares & Frecuencia & \% & IC $\mathbf{9 5 \%}$ \\
& & & \\
\hline & & & \\
Familia extensa & 92 & 38 & $31-44$ \\
Familia nuclear & 130 & 53 & $47-57$ \\
Dos hijos en la familia & 100 & 41 & $34-50$ \\
Parejas en unión libre & 112 & 46 & $39-53$ \\
Vivienda no propia & 90 & 37 & $30-43$ \\
Rol del padre formador de hijos y proveedor económico & 133 & 55 & $48-61$ \\
Ingresos económicos hasta un SMMLV & 115 & 47 & $41-54$ \\
Afiliación al Sistema de Salud a través del régimen subsidiado & 157 & 65 & $59-70$ \\
Antecedentes familiares de Hipertensión & 53 & 22 & $16-27$ \\
Familia presenta problemas de salud oral & 160 & 66 & $60-71$ \\
Visitan al odontólogo por curación & 85 & 35 & $29-41$ \\
Visitan al odontólogo por urgencia & 49 & 20 & $15-25$ \\
Disfuncionalidad familiar leve & 94 & 39 & $33-45$ \\
Disfuncionalidad familiar moderada & 38 & 16 & $11-20$ \\
Disfuncionalidad familiar severa & 5 & 2 & $0,002-0,3$ \\
\hline
\end{tabular}

Tabla 2. Prevalencia de los factores familiares en niños de la institución educativa Madre Gabriela de San Martín, 2010.

\begin{tabular}{lccccc}
\hline Factor Familiar & Caries & No caries & OR & IC=95\% & Valor P \\
\hline Más de 4 hijos & & & & & \\
No nuclear & 12 & 9 & 0,76 & $0,30-1,88$ & 0,55 \\
Vivienda arrendada & 63 & 52 & 0,75 & $0,45-1,24$ & 0,26 \\
Padres separados & 47 & 43 & 0,75 & $0,45-1,27$ & 0,29 \\
Ingresos hasta 1 SMMLV & 23 & 23 & 1,05 & $0,55-1,99$ & 0,87 \\
Estrato socioeconónico bajo & 103 & 108 & 0,49 & $0,22-1,08$ & 0,08 \\
Baja escolaridad padre & 78 & 80 & 0,82 & $0,48-1,40$ & 0,48 \\
Baja escolaridad madre & 29 & 22 & 1,34 & $0,72-2,50$ & 0,34 \\
No presentan Seguridad Social & 15 & 14 & 1,03 & $0,47-2,24$ & 0,93 \\
Otras personas cuida al niño & 46 & 54 & 1,04 & $0,14-7,52$ & 0,96 \\
Presentan antecedentes familiares (hipertensión) & 43 & 37 & 0,84 & $0,49-1,45$ & 0,55 \\
Presentan problemas salud oral & 78 & 65 & 1,40 & $0,84-2,35$ & 0,19 \\
Disfuncionalidad & 82 & 78 & 1,02 & $0,60-1,74$ & 0,92 \\
No visitan al odontólogo & 58 & 59 & 0,81 & $0,48-1,35$ & 0,4 \\
No ejerce el rol el padre & 79 & 70 & 0,81 & $0,48-1,36$ & 0,43 \\
No ejerce el rol la madre & 25 & 11 & 0,40 & $0,18-0,86$ & $0,01^{*}$ \\
\hline
\end{tabular}

Tabla 3. . Asociación de factores socios demográficos y familiares a la prevalencia de caries dental en niños de la institución educativa Madre Gabriela de San Martín. 2010. *Valores de p significativos.

Para la validez de apariencia y la plausibilidad teórica del instrumento, se realizaron dos sesiones de estandarización por los especialistas en Salud Familiar y Odontopediatría de la institución universitaria. Para los resultados de las pruebas de concordancia entre los examinadores clínicos, se realizó un análisis intra e inter-examinador, asumiendo un grado de acuerdo de 0,81, a través de la prueba Kappa Cohen. Para el procesamiento y análisis de la información se utilizaron los programas Microsoft Excel 2007, EPIINFO versión 3.5.1 y
STATA 10.0. Las medidas descriptivas se estimaron a partir de ocurrencias de presencia y severidad de la caries dental y de las variables familiares y demográficas, asumiendo intervalos de confianza del $95 \%$, y para determinar las asociaciones se utilizaron las razones de disparidad (OR).

\section{RESULTADOS}

La tabla 1 muestra la distribución de las características de la población objeto de estudio. 
Entre las variables socio demográficas, las de mayor prevalencia fueron el estrato socioeconómico de nivel dos bajo (47\%; IC 95\%: 40-53), el grado de escolaridad en el padre de secundaria incompleta (38\%; IC 95\%: 31-44) y la unión libre (46\%; IC 95\%: 39-53). Entre las variables familiares se encontró la familia nuclear en un 53\% (IC 95\%: 47-57), el $66 \%$ (IC 95\%; 59-73) estaban afiliadas al sistema de salud, el 66\% (IC 95\%: 60-73) de las familias presentaban problemas de salud bucal y el $35 \%$ (IC 95\%: 48-52) de las familias visitaban al odontólogo por curación (tabla 2).

Se encontró una prevalencia de caries dental del 51\% (IC 95\%: 45-59), siendo mayor para los niños de 8 y 9 años en un $32 \%(p=0,04)$. El tipo de superficie dentaria más comprometida fue la oclusal, en un 40\% (IC 95\%: 2,75-3,52), seguida de la vestibular, en el $38 \%$ (IC 95\%: 2,77-3,41). En cuanto al sexo, se observó una mayor frecuencia en las niñas $(56 \% ; p=0,02)$.

Se encontró únicamente asociación estadísticamente significativa de tipo protector entre la ocurrencia de caries y el rol ejercido por el hombre, padre de familia (OR: 0,40; IC 95\%: 0,18-0,86; $p<0,01)$ (tabla 3).

\section{DISCUSIÓN}

Se puede definir la salud familiar y comunitaria como la "salud individual y de grupos en una comunidad definida, determinada por la interacción de factores personales, familiares, por el ambiente socio-económico-cultural y físico" ${ }^{10}$. En la búsqueda de una adecuada salud bucal de los niños se debe articular la salud de su contexto familiar, puesto que ésta puede influir como protectora o de riesgo para la producción de caries dental según Fejerskov ${ }^{11}$. En este estudio se encontró alta prevalencia de caries dental $(51 \%)$, siendo superior en el sexo femenino, resultados similares a los encontrados por González en 2005 en niños de Cartagena ${ }^{12}$. Además, se encontró que la gran mayoría de estos niños con caries vivían en familias nucleares, aunque no se encontraron asociaciones significativas. Sin embargo, García sugiere que la familia nuclear puede comportarse como un factor protector $^{13}$, contrario a lo reportado por Allan Pau, quien encontró que los niños que vivían con uno de sus dos padres presentaban más dolor dental ${ }^{8}$.
Otro hallazgo de importancia en el presente estudio tiene que ver con el rol del padre, el cual ejercía un rol no sólo de proveedor económico, sino de formador de hijos, presentándose asociación en sentido protector hacia la enfermedad, lo que nos lleva a sugerir que al fortalecer la figura paterna se podría disminuir la aparición de la caries dental, confirmando la reestructuración de los roles paternos y maternos por los cambios que vive hoy en día la sociedad. Este aspecto coincide con lo expresado por Monteverde ${ }^{14}$, donde la mujer está obligada hoy a trabajar por las grandes demandas económicas de una familia y esto provoca que la formación de los hijos sea compartida, evidenciándose un posible impacto en la salud bucal de los niños.

Por otra parte, la mayoría de estos niños vivían en estrato socioeconómico bajo y el $87 \%$ de las familias contaban con ingresos menores a $1 \mathrm{SMMLV}$, lo que se comportaría como riesgo para la enfermedad según Faggiano ${ }^{15}$ y Eckersley $^{16}$, quienes relacionan la presencia de caries dental en dirección inversa al estrato socioeconómico y a los salarios familiares respectivamente. Familias con estas condiciones no dispondrían de recursos suficientes para invertir en su salud oral, tanto en la casa como en el acceso al servicio de salud odontológico. Igual sucede con el bajo nivel de escolaridad de las madres, que ha estado relacionado con la aparición de caries dental3,4,12. En el presente estudio el nivel de escolaridad de los padres de los niños con caries fue la secundaria incompleta, pero esta no fue estadísticamente significativa. Todos estos factores contribuyen a aumentar la pobreza en estos hogares y a volverlos vulnerables ante la enfermedad. Esto sin contar el hecho de que no contar con servicios de salud representa un riesgo para la salud bucal según Pilar Amaya ${ }^{17}$. Casi todas las familias de los escolares se encontraban afiliadas al Sistema de Seguridad Social bajo el régimen subsidiado, pero no es coherente con los resultados encontrados y no se observó relación significativa. En este sentido, pueden surgir hipótesis como el poco uso de los servicios de salud, baja cobertura, mala calidad de los mismos o pocas actividades de promoción y prevención en salud bucal.

Los resultados del presente estudio indican que algunos aspectos asociados a la familia pueden actuar como factores influyentes, siendo el rol de 
los padres el que presenta significancia estadística. Por lo tanto, se debe plantear un acompañamiento familiar en los actuales programas de promoción y prevención en salud bucal para lograr impacto en estas acciones.

\section{BIBLIOGRAFÍA}

1. Romero M, Bedregal P, Bastías G. Situación Salud Materno infantil y familia en Chile. Boletín Escuela de Medicina, $P$ Universidad Católica de Chile. 1994; 23:10-4.

2. Arnrup K, Broberg A, Lundin S, Hakeberg M. Attitudes to dental care among parents of uncooperative vs. cooperative child dental patients. Europe Journal Oral Sciences. 2002; 110:75-82.

3. Pérez S, Soto L. Caries dental en primeros molares permanentes y factores socioeconómicos en escolares de Campeche, México. Revista Cubana Estomatología. 2002; 39(3):265-81.

4. Franco AS, Kurzer E, Castro L, Giraldo M. El menor de seis años: Situación de caries y conocimientos y prácticas de cuidado bucal de sus madres. Revista CES Odontología. 2004; 17(1):19-29.

5. Bustamante Z, Camargo L, Franco A. Estado de salud bucal de niños preescolares y escolares de nivel socioeconómico alto y medio alto. Revista CES Odontología. 1998; 11(1):358.

6. Martignon S. Caries dental y asociación a factores de riesgo en la población escolar de Moniquirá, Boyacá. Revista científica Universidad del Bosque 2003; 9(2):28-34

7. Gonzalvo GO. Indicadores de Maltrato Infantil. Guías Clínicas de España 2002; 2(44).

8. Pau A, Khan S, Babar M, Croucher R. Dental pain and care-seeking in 11-14-yr-old adolescents in a low-income country. Europe Journal Oral Science. 2008; 116:451-7.
9. González, F. Carmona, L. Pérez, G. Prevalencia de caries dental "Ekstrand" y factores de riesgo en niños escolarizados con edades entre 5 y 13 años del barrio Nelson Mándela de la Ciudad de Cartagena de indias. 2004. Memorias XVI Encuentro Nacional de Investigación Odontológica Universidad El Bosque- ACFO Bogotá; 2004. p. 24.

10. Gofin J, Gofin R. Essentials of global community health. Sudbury, MA: Jones \& Barlett Learning; 2010. p. 269.

11. Fejerskov O. Changing paradigms in concepts on Dental Caries: Consequences for Oral Health Care. Caries Research. 2004; 38:182-91.

12. González F, Luna L, Martínez N, Solana M. Correlación entre los factores de riesgo biológicos y sociales con la presencia de caries dental en niños entre 5 y 6 años de cinco hogares infantiles del Instituto Colombiano de Bienestar familiar de la Cuidad de Cartagena, 2005-2006. Federación Odontológica Colombiana. 2007; 69: 7-20.

13. García-Campayo J, Alda M. Familias con características especiales. En: García-Campayo J, editor. La familia y el médico de familia: elementos básicos de intervención desde atención primaria. Madrid: Mayo; 2004. p. 37-44.

14. Monteverde GG. ¿Por qué trabajan las mujeres? Revista El Colegio de Sonora. 1996; 7(12):161-7 [consultado el 10 de mayo de 2007]. Disponible en: http://lanic.utexas.edu/ project/etext/colson/12/12 6.pdf.

15. Faggiano F, Stanislao FD, Lemma P, Renga G. Role of social class in caries occurrence in 12 year olds in Turin, Italy. Eur J Public Health. 1999; 9:109-13.

16. Eckersley AJ, Blinkhorn FA. Dental attendance and dental health behavior in children from deprived and non-deprived areas of Salford, north-west England. Int J Paediatr Dent. 2001; 11:103-9.

17. Amaya P. Instrumento de riesgo familiar total RFT: 5-33. Manual. Aspectos Teóricos, psicométricos, de estandarización y de aplicación del instrumento. Universidad Nacional de Colombia; 2004. 